\title{
Lattice QCD Equation of State at Finite Chemical Potential from an Alternative Expansion Scheme
}

\author{
S. Borsányi®, ${ }^{1}$ Z. Fodor, ${ }^{2,1,3,4}$ J. N. Guenther, ${ }^{5}$ R. Kara $\odot,{ }^{1}$ S. D. Katz, ${ }^{6}$ P. Parotto $\odot,{ }^{1}$ \\ A. Pásztorø ${ }^{6}$ C. Ratti, ${ }^{7}$ and K. K. Szabó ${ }^{1,4}$ \\ ${ }^{1}$ University of Wuppertal, Department of Physics, Wuppertal D-42119, Germany \\ ${ }^{2}$ Pennsylvania State University, Department of Physics, State College, Pennsylvania 16801, USA \\ ${ }^{3}$ Institute for Theoretical Physics, ELTE Eötvös Loránd University, Pázmány Péter sétány 1/A, H-1117 Budapest, Hungary \\ ${ }^{4}$ Jülich Supercomputing Centre, Forschungszentrum Jülich, D-52425 Jülich, Germany \\ ${ }^{5}$ Aix Marseille Université, Université de Toulon, CNRS, CPT, Marseille, France \\ ${ }^{6}$ Eötvös University, Budapest 1117, Hungary \\ ${ }^{7}$ Department of Physics, University of Houston, Houston, Texas 77204, USA
}

(Received 31 March 2021; accepted 13 May 2021; published 11 June 2021)

\begin{abstract}
In this Letter, we introduce a novel scheme for extrapolating the equation of state of QCD to finite chemical potential that features considerably improved convergence properties and allows us to extend its reach to unprecedentedly high baryonic chemical potentials. We present continuum extrapolated lattice results for the new expansion coefficients and show the thermodynamic observables up to $\mu_{B} / T \leq 3.5$. This novel expansion does not suffer from the shortcomings that characterize the traditional Taylor expansion method, such as difficulties inherent in performing such an expansion with a limited number of coefficients and the poor signal-to-noise ratio that affects Taylor coefficients determined from lattice calculations.
\end{abstract}

DOI: 10.1103/PhysRevLett.126.232001

Introduction.-The phase diagram of quantum chromodynamics (QCD) is an open field of investigation, which is at the center of intense efforts from the theoretical and experimental communities. At vanishing baryon density, first principle results show that the transition between confined and deconfined matter is an analytic crossover [1]. Although at finite baryon density, lattice QCD faces a sign problem, numerous results have been published for moderate chemical potentials [2,3]. New techniques that allow direct simulations at finite chemical potential in the presence of a sign problem include Lefschetz thimbles [4-6], the Complex Langevin equation [7-10] or reweighting-based methods [11]. These approaches cannot be applied to large scale QCD simulations yet.

The most straightforward method for studying QCD at finite density is the Taylor expansion, where the leading $\mu_{B}$ derivatives of the relevant observables are calculated [1215]. These derivatives were often calculated for chiral observables, and the $\mu_{B}$ dependence of the transition temperature was extracted [16-19]. These coefficients can be efficiently calculated by simulating imaginary values of the chemical potential(s), besides $\mu_{B}=0$

Published by the American Physical Society under the terms of the Creative Commons Attribution 4.0 International license. Further distribution of this work must maintain attribution to the author(s) and the published article's title, journal citation, and DOI. Funded by SCOAP ${ }^{3}$.
[20,21]. This is motivated by the analytic crossover at $\mu_{B}=0$, from which the smooth behavior of the thermodynamic observables as a function of $\mu_{B}^{2}$ follows [18, 22-27]. This is often referred to as analytical continuation. This name suggests that, besides the computation of the Taylor expansion coefficients, other extrapolation schemes can be established. For example, the Padé summation was also considered in the context of QCD thermodynamics [28-32]. The success of this method was most visible in the study of the QCD transition line, where continuum extrapolated results are available for the leading $\mu_{B}$ dependence [18,33-35] and, recently, also for the nextto-leading coefficient [36].

The knowledge of the QCD phase diagram from lattice simulations is currently limited to small $\mu_{B}$, and data are mostly available in the transition region. We have to mention that, at high temperatures, resummed perturbation theory has provided a quantitative description of the chemical potential dependence of several observables [37-39]. Dedicated lattice studies have bridged the gap between the transition region and perturbative temperatures and found perfect agreement [40,41].

On the experimental side, heavy-ion collisions are mapping out the phase structure of strongly interacting matter. The evolution of the system created in these experiments can be described by hydrodynamic simulations, which need the equation of state of QCD as an input in the whole range of temperatures and densities covered in the experiments. Recently, a Bayesian analysis based on a 
systematic comparison between heavy-ion data and theoretical predictions showed that the posterior distribution over possible equations of states is compatible with the one calculated on the lattice [42]. For this reason, the equation of state at finite density is a crucial ingredient for supporting the experimental program.

The equation of state at vanishing chemical potential has been known, now, for several years over a broad range of temperatures [43-45]. The first continuum extrapolated extension to finite $\mu_{B}$ using the Taylor method in Ref. [14] was followed by several works with the intent of extending these results to higher $\mu_{B}$ by adding more terms in the Taylor series [15,46,47]. Currently, even the sixth $\mu_{B}$ derivative of the QCD pressure is available with modest precision from lattice simulations $[21,47]$. Recently, similar results were found by solving a QCD-assisted effective theory with functional methods [48].

In this Letter, we propose a new scheme to extrapolate the equation of state of QCD to finite density. We intend to remedy some shortcomings of the Taylor-based equation of state, e.g., the extrapolation through a crossover boundary, as detailed below. We will show that this expansion converges faster than the Taylor series at finite density, thus, leading to an unprecedented coverage in $\mu_{B}$ and to more precise results for the thermodynamic observables.

Motivation and methodology.-The knowledge of the equation of state from lattice simulations commonly consists of the established $\mu_{B}=0$ result $[44,45]$ and the Taylor expansion coefficients of the pressure around $\mu_{B}=0$

$$
\frac{p\left(T, \mu_{B}\right)}{T^{4}}=\sum_{n=0} \frac{1}{(2 n) !} \chi_{2 n}^{B}(T, 0)\left(\frac{\mu_{B}}{T}\right)^{2 n},
$$

where $\chi_{j}^{B}$ are the $j$ th derivatives of the normalized pressure

$$
\chi_{j}^{B}\left(T, \mu_{B}\right)=\left(\frac{\partial}{\partial \mu_{B} / T}\right)^{j} \frac{p\left(T, \mu_{B}\right)}{T^{4}} .
$$

Besides diagonal coefficients, one can also define offdiagonal correlators between different conserved charges in QCD. Correlators between baryon number and strangeness are defined as follows:

$$
\chi_{j k}^{B S}\left(T, \mu_{B}\right)=\left(\frac{\partial}{\partial \mu_{B} / T}\right)^{j}\left(\frac{\partial}{\partial \mu_{S} / T}\right)^{k} \frac{p\left(T, \mu_{B}\right)}{T^{4}} .
$$

Such correlators have phenomenological relevance [49] and they can also be used to extrapolate the equation of state of QCD in the full, four-dimensional phase diagram at finite $T, \mu_{B}, \mu_{S}, \mu_{Q}[50,51]$. We will use the $\hat{\mu}_{i}=\mu_{i} / T$ shorthand notation in this manuscript. Currently, results for the expansion coefficients are available up to order $\mathcal{O}\left(\mu_{B}^{6}\right)$ $[21,47]$. The region of validity of the resulting expansion is usually determined by the range in chemical potential within which an apparent convergence is achieved. This is stated to be $\hat{\mu}_{B} \lesssim 2-2.5[15,46]$.

High order derivatives of the pressure are notoriously difficult to calculate, as they suffer from a low signal-tonoise ratio [40]. Moreover, studies of chiral models revealed that the structure of the temperature dependence of such observables becomes more and more complex when higher orders are considered [52]. This may explain why including one more term in a truncated Taylor series will not always improve the convergence. On the contrary, pathological behavior-namely, nonmonotonicity in the $T$ or $\mu_{B}$ dependence-appears in the extrapolated thermodynamic quantities at chemical potentials beyond $\hat{\mu}_{B} \lesssim 2-2.5$. Such an effect in the truncated Taylor series was pointed out, e.g., in Refs. [53-55]. This is due to the fact that, for large enough $\hat{\mu}_{B}$, the observables at finite chemical potential are dictated by the $\mu_{B}=0$ temperature dependence of the last coefficient included in the expansion. Hence, the structures appearing around the QCD transition temperature in higher order coefficients are "translated" into the finite- $\mu_{B}$ behavior of, e.g., the entropy, baryon density, etc. Another inherent problem with the Taylor expansion is the fact that it is carried out at constant temperature. This means that the values of the coefficients at $\mu_{B}=0$ and a certain temperature $T$, determine the equation of state at the same $T$ at finite $\mu_{B}$, while the pseudocritical temperature $T_{p c}$ might have varied considerably.

In Fig. 1, we show the baryon density $n_{B}(T)$ obtained from a Taylor expansion with the coefficients in Ref. [21], at $\hat{\mu}_{B}=3$. The extrapolation is shown including an increasing number of coefficients, to show the effect of higher-order ones. The leading-order and higher truncations refer to $\sim \hat{\mu}_{B} \partial n_{B}(T) / \partial \hat{\mu}_{B}$, or $\sim \frac{1}{6} \hat{\mu}_{B}^{3} \partial^{3} n_{B}(T) / \partial \hat{\mu}_{B}^{3}$, etc. being the last term in the expansion. The derivatives are

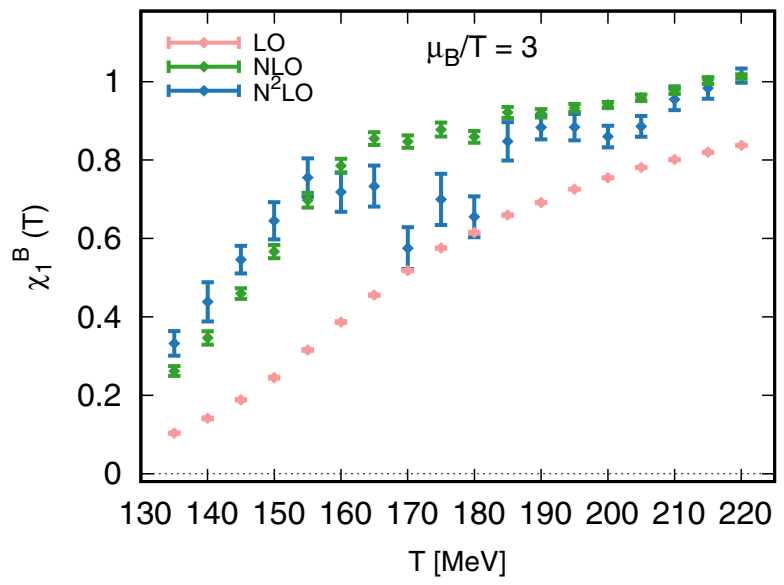

FIG. 1. Baryon density from a Taylor expansion with the coefficients in Ref. [21], at $\mu_{B} / T=3$, as a function of the temperature. Different colors correspond to the order to which the expansion is carried out: leading order (LO) in pink, next-toleading order (NLO) in green, and next-to-next-to-leading order (N2LO) in blue. 
taken at $\mu_{B}=0$. Two features emerge from Fig. 1: (1) the LO and next-to-leading order datasets are very different from each other, meaning that the series is not converging fast at this $\hat{\mu}_{B} ;$; (2) the inclusion of all the coefficients in Ref. [21] causes unphysical nonmonotonic behavior. Incidentally, recent estimates on coarse lattices [56,57], but also universality arguments [58], place the convergence in the same ball park in $\mu_{B}$.

Here, we present an alternative summation scheme which can better cope with the fact that the QCD transition temperature presents a $\mu_{B}$ dependence. We start from the observation that we made while working with imaginary values of the chemical potentials in an earlier work. In the upper panel of Fig. 2, we show temperature scans of the quantity $n_{B}(T) / \hat{\mu}_{B}=\chi_{1}^{B}\left(T, \hat{\mu}_{B}\right) / \hat{\mu}_{B}$ for several fixed imaginary $\hat{\mu}_{B}$ values. The $0 / 0$ limit at $\mu_{B}=0$ can be easily resolved and equals $\chi_{2}^{B}(T)$.

The $T$ dependence of the normalized baryon density at finite chemical potential appears to be simply rescaled
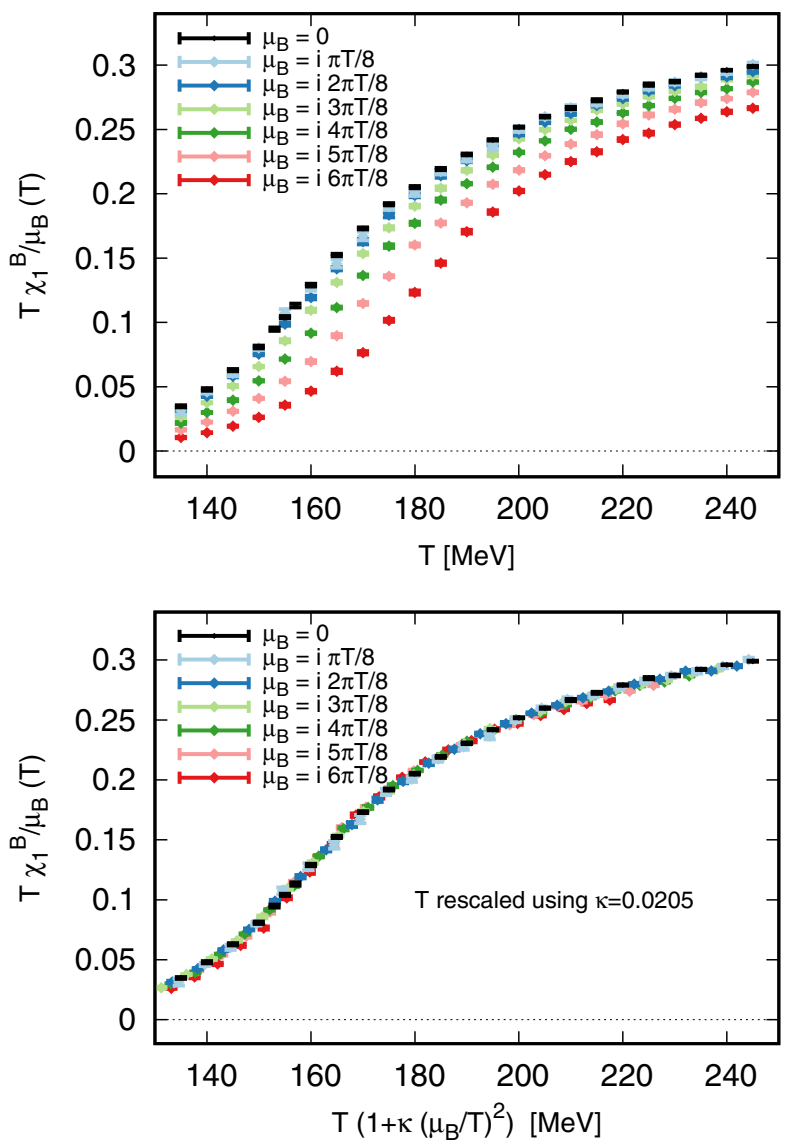

FIG. 2. Upper panel: The (imaginary) baryon density at simulated (imaginary) baryon chemical potentials, divided by the chemical potential. The points at $\mu_{B}=0$ (black) show the second baryon susceptibility $\chi_{2}^{B}(T)$. Lower panel: same curves as in the upper panel, with a temperature rescaled in accordance to Eq. (5) with $\kappa=0.0205$. toward higher temperatures from the $\mu_{B}=0$ results for $\chi_{2}^{B}$. A simple rescaling of temperatures can be described as

$$
\frac{\chi_{1}^{B}\left(T, \hat{\mu}_{B}\right)}{\hat{\mu}_{B}}=\chi_{2}^{B}\left(T^{\prime}, 0\right)
$$

where the actual temperature difference can be expressed through a $\mu_{B}$-dependent factor that we write, for simplicity, as

$$
T^{\prime}=T\left(1+\kappa \hat{\mu}_{B}^{2}\right) .
$$

In the lower panel of Fig. 2, we show a version of the curves in the upper panel, with the finite- $\hat{\mu}_{B}$ curves rescaled following Eq. (5) with $\kappa=0.0205$. We note how well the curves are superimposed to each other, even assuming a single, $T$-independent parameter governing the transformation.

Although rather suggestive, the description following from Eq. (5) cannot serve as an alternative expansion scheme. To this end, first, we note that, at vanishing chemical potential, we can express the normalized baryon density as a Taylor expansion

$\frac{\chi_{1}^{B}}{\hat{\mu}_{B}}\left(T, \hat{\mu}_{B}\right)=\chi_{2}^{B}(T, 0)+\frac{\hat{\mu}_{B}^{2}}{6} \chi_{4}^{B}(T, 0)+\frac{\hat{\mu}_{B}^{4}}{120} \chi_{6}^{B}(T, 0)+\cdots$.

Then, we systematically generalize Eq. (5) assuming an expansion in $T^{\prime}$ with temperature-dependent coefficients

$T^{\prime}\left(T, \hat{\mu}_{B}\right)=T\left[1+\kappa_{2}^{B B}(T) \hat{\mu}_{B}^{2}+\kappa_{4}^{B B}(T) \hat{\mu}_{B}^{4}+\mathcal{O}\left(\hat{\mu}_{B}^{6}\right)\right]$.

In the above equation, we introduced the new parameters $\kappa_{2}^{B B}(T)$ and $\kappa_{4}^{B B}(T)$, which describe the rescaling of the temperature of $\chi_{1}^{B} / \hat{\mu}_{B}$ at finite $\mu_{B}$.

Now, having two expressions, Eqs. (6) and (4), for the same quantity, we require their equality at each order in the $\hat{\mu}_{B}$ expansion at $\mu_{B}=0$, having

$$
\begin{aligned}
& \chi_{4}^{B}(T)=6 T \kappa_{2}^{B B}(T) \frac{d \chi_{2}}{d T}, \\
& \chi_{6}^{B}(T)=60 T^{2}\left(\kappa_{2}^{B B}\right)^{2}(T) \frac{d^{2} \chi_{2}}{d T^{2}}+120 T \kappa_{4}^{B B}(T) \frac{d \chi_{2}}{d T},
\end{aligned}
$$

which, in turn, yields

$$
\begin{aligned}
\kappa_{2}^{B B}(T) & =\frac{1}{6 T} \frac{\chi_{4}^{B}(T)}{\chi_{2}^{B \prime}(T)}, \\
\kappa_{4}^{B B}(T) & =\frac{1}{360 \chi_{2}^{B \prime}(T)^{3}}\left[3 \chi_{2}^{B \prime}(T)^{2} \chi_{6}^{B}(T)-5 \chi_{2}^{B \prime \prime}(T) \chi_{4}^{B}(T)^{2}\right] .
\end{aligned}
$$

This construction amounts to a reorganization of the "canonical" Taylor series, wherein the systematic 
expansion is carried in the quantity $\left(T^{\prime}-T\right) / T$. Essentially, this formalism replaces the fixed-temperature $\hat{\mu}_{B}$ expansion by a fixed-observable temperature expansion. We note that observations and definitions analogous to those just summarized can be made for strangeness-related quantities, too, as described in the Supplemental Material [59], together with the details of our formalism.

We remark that very similar equations have already been used in Ref. [15] to calculate "lines of constant physics" to $\mathcal{O}\left(\mu_{B}^{4}\right)$ order. In this reference, the pressure, energy density, and entropy were calculated using the Taylor method, and in a further step, lines were drawn on the $\mu_{B}-T$ phase diagram, where these quantities are constant in some normalization. The obtained $\kappa_{2}$ coefficients are closely related to ours. Contrary to Ref. [15], we use Eq. (7) as the definition of a truncation scheme rather than to investigate a Taylor expanded result.

Starting from the results at imaginary chemical potentials in Fig. 2, we base our description of the entire chemical potential dependence of the QCD free energy function on $\chi_{1}^{B}\left(T, \hat{\mu}_{B}\right) / \hat{\mu}_{B}$ and Eq. (5). It is essential that the truncation scheme is based on one observable only, in order to guarantee thermodynamic consistency: then, other quantities will automatically obey thermodynamic relations. Alternatively, one could base the procedure on the pressure, entropy, or energy density, but the baryon density $\chi_{1}^{B}$ proves to be the better choice due to its simplicity and the better signal-to-noise ratio.

Results.-For the determination of $\kappa_{2}^{B B}$ and $\kappa_{4}^{B B}$, one can take advantage of simulations both at zero and finite imaginary chemical potential. First, we calculated $\kappa_{2}^{B B}(T)$ using Eq. (9). To extract $\kappa_{4}^{B B}(T)$ using the same strategy, a precise result on $\chi_{6}^{B}(T)$ would be necessary.

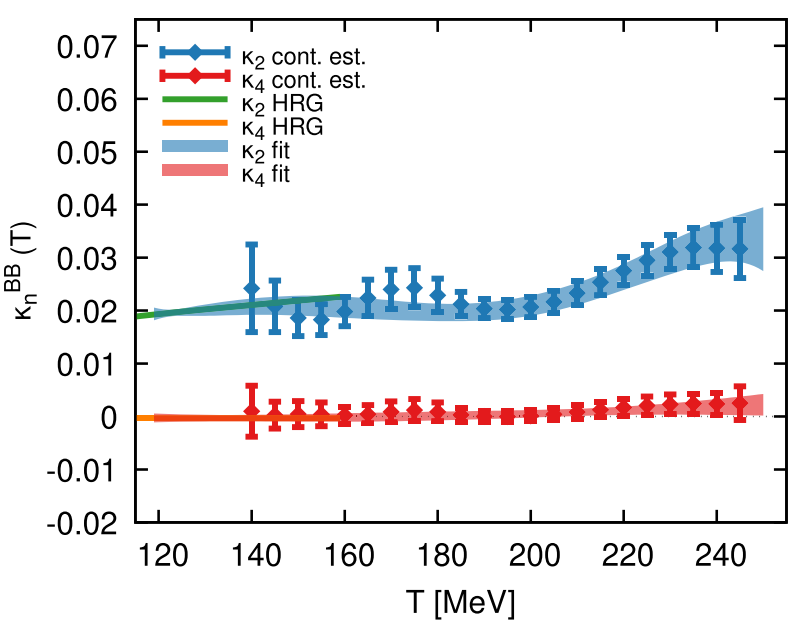

FIG. 3. Continuum extrapolated result for the expansion parameters $\kappa_{2}^{B B}(T)$ and $\kappa_{4}^{B B}(T)$ (top panel). HRG results are shown up to $T=160 \mathrm{MeV}$ (in green for $\kappa_{2}^{B B}$ and orange for $\kappa_{4}^{B B}$, respectively). The bands show correlated polynomial fits as described in the text.
Instead, we utilize imaginary chemical potential simulations, as detailed in the Supplemental Material [59].

In Fig. 3, we show the results of the temperature-bytemperature fit procedure for the parameters $\kappa_{2}^{B B}(T)$ and $\kappa_{4}^{B B}(T)$, along with the hadron resonance gas (HRG) model results. We find that, within errors, $\kappa_{2}^{B B}(T)$ has hardly any dependence on the temperature, while $\kappa_{4}^{B B}(T)$ is everywhere consistent with zero at our current level of precision. Nonetheless, a clear separation of almost 1 order of magnitude appears between these two coefficients. We also note that good agreement with the HRG results is found up to at least $T=150 \mathrm{MeV}$. Notably, a clear scale separation between analogous $\kappa_{2}$ and $\kappa_{4}$ parameters at the pseudocritical temperature is observed in chiral observables, too $[19,36]$, as well as in the strangeness-related observables discussed in the Supplemental Material [59].

In order to limit the influence of numerical effects on the final observables, we construct smoother versions of our final results for $\kappa_{2}^{B B}$ and $\kappa_{4}^{B B}$, which are shown in Fig. 3 as transparent bands. Because of the mild $T$ dependence, we perform a polynomial fit of order 5 for $\kappa_{2}^{B B}$, and of order 2 for $\kappa_{4}^{B B}$. The very good fit qualities show no need for higher order polynomials. In order to stabilize the low-temperature behavior, we included in the fit two points from the HRG model. The fits fully take into account the systematic as well as statistical correlations between different temperatures. The results of the fit are used as the input in the thermodynamic calculations that follow.

From Eq. (5), $n_{B}$ can be determined at finite real chemical potential and, from it, the other thermodynamic quantities. The integration constant for the pressure is obviously the pressure itself at $\mu_{B}=0$. The thermodynamic relationships that we use to generate all relevant observables are detailed in the Supplemental Material [59]. We present our results for the finite real chemical potential extrapolation of several thermodynamic quantities: the various panels of Fig. 4 show the baryon density, pressure, entropy, and energy density for $\hat{\mu}_{B}=0-3.5$. Alongside our results, we show predictions from the HRG model for $T<150 \mathrm{MeV}$, which are in very good agreement with our extrapolation for all observables, at all values of the chemical potential. We note that the observables do not suffer from the pathological behavior that affects the Taylor expansion, thus, highly improving the results currently available in the literature.

In the upper left panel of Fig. 4, we also show the comparison of our results for the baryon density to the simplified case where $\kappa_{4}^{B B}$ is neglected. The inclusion of the next-to-leading-order parameter came at the cost of an increased uncertainty at larger chemical potential. However, the results are compatible with each other, which demonstrates the improved convergence of our method.

Conclusions. - We proposed an alternative summation scheme for the equation of state of QCD at finite real chemical potential, designed to overcome the typical 

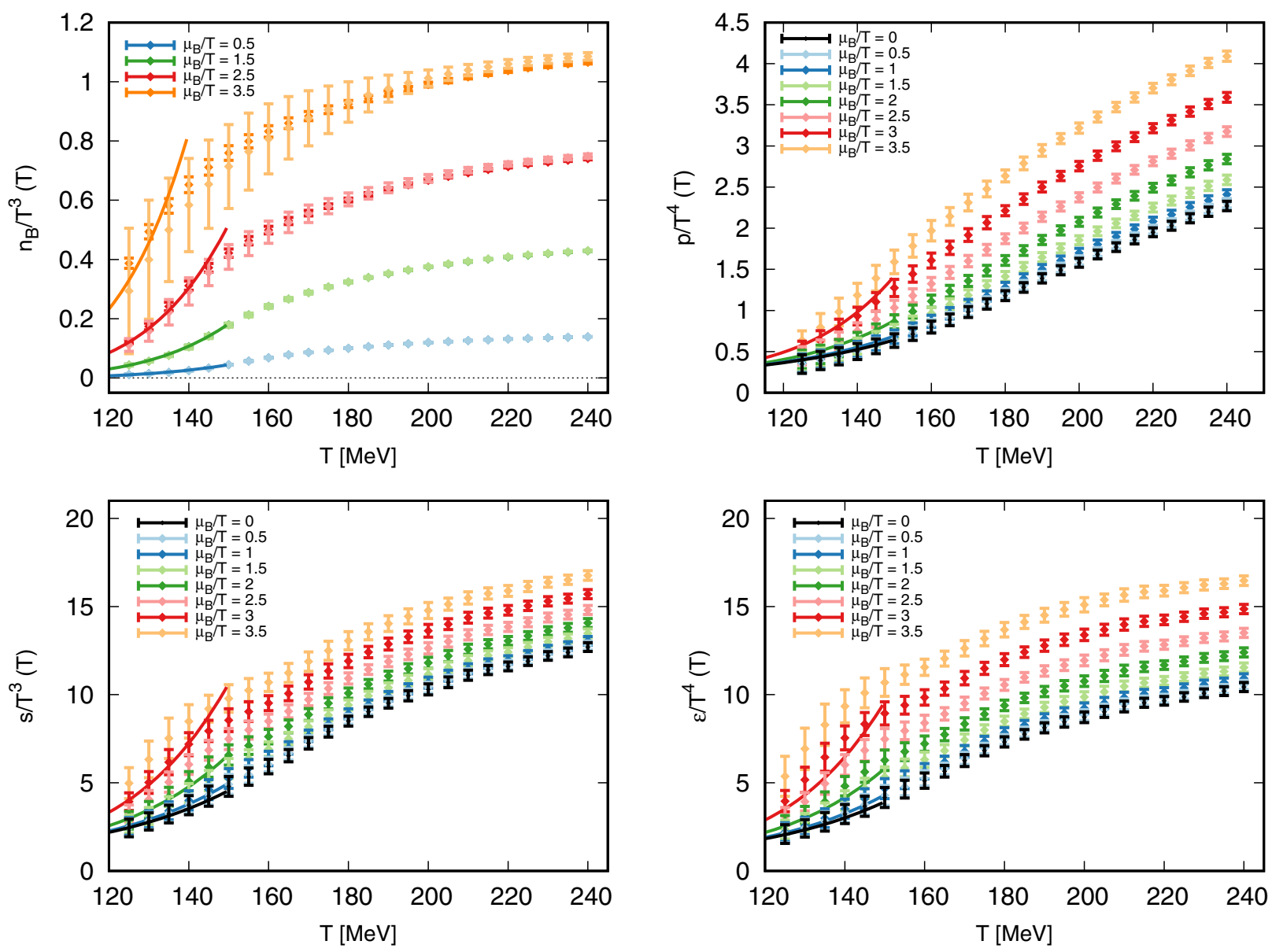

FIG. 4. Baryon density, pressure, entropy, and energy density at increasing values of $\hat{\mu}_{B}$. With solid lines, we show the results from the HRG model. For the baryon density (top left panel) we also show, in darker shades, the results obtained by omitting the parameter $\kappa_{4}^{B B}$.

shortcomings of the Taylor expansion. Through simulations at zero and imaginary chemical potentials, we determined the LO and NLO parameters describing the chemical potential dependence of the baryon density, which we, then, extrapolated to large real chemical potentials.

By combining this new element, and previously published results for the equation of state at vanishing $\mu_{B}$, we reconstructed all thermodynamic variables at chemical potential as large as $\mu_{B} / T=3.5$ with rather limited uncertainty. Systematic as well as statistical errors were considered in the analysis.

Our results, although still limited in precision at the level of $\kappa_{2}^{B B}$ and $\kappa_{4}^{B B}$, suggest that the avenue we pursue in this Letter is rather promising for the description of QCD thermodynamics at finite chemical potential. Moreover, our procedure is systematically improvable with sufficient computing power, and might prove to be a better strategy than existing canonical approaches.

In this Letter, we limited ourselves to the case where the strange and electric chemical potentials are set to zero. We reserve, for future work, the exploration of the phenomenologically relevant case of strangeness neutrality and fixed electric charge to baryon ratio.
This project was funded by the DFG Grant No. SFB/ TR55. The project also received support from the BMBF Grant No. 05P18PXFCA. This work was also supported by the Hungarian National Research, Development and Innovation Office, NKFIH Grant No. KKP126769. A.P. is supported by the J. Bolyai Research Scholarship of the Hungarian Academy of Sciences and by the ÚNKP-20-5 New National Excellence Program of the Ministry for Innovation and Technology. The project leading to this publication has received funding from the Excellence Initiative of Aix-Marseille University- $A *$ MIDEX, a French "Investissements d'Avenir" programme, Grant No. AMX-18-ACE-005. This material is based upon work supported by the National Science Foundation under Grant No. PHY-1654219 and by the U.S. DOE, Office of Science, Office of Nuclear Physics, within the framework of the Beam Energy Scan Topical (BEST) Collaboration. This research used resources of the Oak Ridge Leadership Computing Facility, which is a DOE Office of Science User Facility supported under Contract No. DE-AC0500OR22725. The authors gratefully acknowledge the Gauss Centre for Supercomputing e.V. [60] for funding this project by providing computing time on the GCS 
Supercomputer HAWK at HLRS, Stuttgart. Part of the computation was performed on the QPACE3 funded by the DFG ind hosted by JSC. C. R. also acknowledges the support from the Center of Advanced Computing and Data Systems at the University of Houston.

[1] Y. Aoki, G. Endrodi, Z. Fodor, S. D. Katz, and K. K. Szabo, The order of the quantum chromodynamics transition predicted by the standard model of particle physics, Nature (London) 443, 675 (2006).

[2] J. N. Guenther, Overview of the QCD phase diagramRecent progress from the lattice, Eur. Phys. J. A 57, 136 (2021).

[3] C. Ratti, Lattice QCD and heavy ion collisions: A review of recent progress, Rep. Prog. Phys. 81, 084301 (2018).

[4] M. Cristoforetti, F. Di Renzo, and L. Scorzato (Aurora Science Collaboration), New approach to the sign problem in quantum field theories: High density QCD on a Lefschetz thimble, Phys. Rev. D 86, 074506 (2012).

[5] A. Alexandru, G. Basar, and P. Bedaque, Monte Carlo algorithm for simulating fermions on Lefschetz thimbles, Phys. Rev. D 93, 014504 (2016).

[6] M. Fukuma, N. Matsumoto, and N. Umeda, Implementation of the HMC algorithm on the tempered Lefschetz thimble method, arXiv:1912.13303.

[7] G. Aarts, F. A. James, J. M. Pawlowski, E. Seiler, D. Sexty, and I.-O. Stamatescu, Stability of complex Langevin dynamics in effective models, J. High Energy Phys. 03 (2013) 073.

[8] D. Sexty, Simulating full QCD at nonzero density using the complex Langevin equation, Phys. Lett. B 729, 108 (2014).

[9] M. Scherzer, E. Seiler, D. Sexty, and I.-O. Stamatescu, Controlling complex Langevin simulations of lattice models by boundary term analysis, Phys. Rev. D 101, 014501 (2020).

[10] D. Sexty, Calculating the equation of state of dense quarkgluon plasma using the complex Langevin equation, Phys. Rev. D 100, 074503 (2019).

[11] M. Giordano, K. Kapas, S. D. Katz, D. Nogradi, and A. Pasztor, New approach to lattice QCD at finite density; results for the critical end point on coarse lattices, J. High Energy Phys. 05 (2020) 088.

[12] C. R. Allton, S. Ejiri, S. J. Hands, O. Kaczmarek, F. Karsch, E. Laermann, C. Schmidt, and L. Scorzato, The QCD thermal phase transition in the presence of a small chemical potential, Phys. Rev. D 66, 074507 (2002).

[13] C. R. Allton, M. Doring, S. Ejiri, S. J. Hands, O. Kaczmarek, F. Karsch, E. Laermann, and K. Redlich, Thermodynamics of two flavor QCD to sixth order in quark chemical potential, Phys. Rev. D 71, 054508 (2005).

[14] S. Borsanyi, G. Endrodi, Z. Fodor, S. D. Katz, S. Krieg, C. Ratti, and K. K. Szabo, QCD equation of state at nonzero chemical potential: Continuum results with physical quark masses at order $m u^{2}$, J. High Energy Phys. 08 (2012) 053.

[15] A. Bazavov et al., The QCD equation of state to $\mathcal{O}\left(\mu_{B}^{6}\right)$ from lattice QCD, Phys. Rev. D 95, 054504 (2017).

[16] O. Kaczmarek, F. Karsch, E. Laermann, C. Miao, S. Mukherjee, P. Petreczky, C. Schmidt, W. Soeldner, and
W. Unger, Phase boundary for the chiral transition in $(2+1)$ -flavor QCD at small values of the chemical potential, Phys. Rev. D 83, 014504 (2011).

[17] G. Endrodi, Z. Fodor, S. D. Katz, and K. K. Szabo, The QCD phase diagram at nonzero quark density, J. High Energy Phys. 04 (2011) 001.

[18] C. Bonati, M. D’Elia, F. Negro, F. Sanfilippo, and K. Zambello, Curvature of the pseudocritical line in QCD: Taylor expansion matches analytic continuation, Phys. Rev. D 98, 054510 (2018).

[19] A. Bazavov et al. (HotQCD Collaboration), Chiral crossover in QCD at zero and non-zero chemical potentials, Phys. Lett. B 795, 15 (2019).

[20] M. D'Elia, G. Gagliardi, and F. Sanfilippo, Higher order quark number fluctuations via imaginary chemical potentials in $N_{f}=2+1$ QCD, Phys. Rev. D 95, 094503 (2017).

[21] S. Borsanyi, Z. Fodor, J. N. Guenther, S. K. Katz, K. K. Szabo, A. Pasztor, I. Portillo, and C. Ratti, Higher order fluctuations and correlations of conserved charges from lattice QCD, J. High Energy Phys. 10 (2018) 205.

[22] P. de Forcrand and O. Philipsen, The QCD phase diagram for small densities from imaginary chemical potential, Nucl. Phys. B642, 290 (2002).

[23] M. D'Elia and M.-P. Lombardo, Finite density QCD via imaginary chemical potential, Phys. Rev. D 67, 014505 (2003).

[24] M. D'Elia, F. Di Renzo, and M. P. Lombardo, The strongly interacting quark gluon plasma, and the critical behavior of QCD at imaginary $\mu$, Phys. Rev. D 76, 114509 (2007).

[25] P. Cea, L. Cosmai, M. D’Elia, C. Manneschi, and A. Papa, Analytic continuation of the critical line: Suggestions for QCD, Phys. Rev. D 80, 034501 (2009).

[26] P. Cea, L. Cosmai, M. D’Elia, C. Manneschi, and A. Papa, On the analytic continuation of the critical line, Proc. Sci., LAT2009 (2009) 161 [arXiv:1001.4439].

[27] C. Bonati, M. D'Elia, P. de Forcrand, O. Philipsen, and F. Sanfilippo, The chiral phase transition for two-flavour QCD at imaginary and zero chemical potential, Proc. Sci., LATTICE2013 (2014) 219 [arXiv:1311.0473].

[28] F. Karsch, B.-J. Schaefer, M. Wagner, and J. Wambach, Towards finite density QCD with Taylor expansions, Phys. Lett. B 698, 256 (2011).

[29] P. Cea, L. Cosmai, M. D'Elia, A. Papa, and F. Sanfilippo, The critical line of two-flavor QCD at finite isospin or baryon densities from imaginary chemical potentials, Phys. Rev. D 85, 094512 (2012).

[30] S. Datta, R. V. Gavai, and S. Gupta, Quark number susceptibilities and equation of state at finite chemical potential in staggered QCD with $N_{t}=8$, Phys. Rev. D 95, 054512 (2017).

[31] A. Pásztor, Z. Szép, and G. Markó, Apparent convergence of Padé approximants for the crossover line in finite density QCD, Phys. Rev. D 103, 034511 (2021).

[32] C. Schmidt, J. Goswami, G. Nicotra, F. Ziesché, P. Dimopoulos, F. Di Renzo, S. Singh, and K. Zambello, Net-baryon number fluctuations, in Criticality in QCD and the Hadron Resonance Gas (2021) [arXiv: 2101.02254]. 
[33] C. Bonati, M. D’Elia, M. Mariti, M. Mesiti, F. Negro, and F. Sanfilippo, Curvature of the chiral pseudocritical line in QCD: Continuum extrapolated results, Phys. Rev. D 92, 054503 (2015).

[34] P. Cea, L. Cosmai, and A. Papa, Critical line of $2+1$ flavor QCD: Toward the continuum limit, Phys. Rev. D 93, 014507 (2016).

[35] R. Bellwied, S. Borsanyi, Z. Fodor, J. Guenther, S. D. Katz, C. Ratti, and K. K. Szabo, The QCD phase diagram from analytic continuation, Phys. Lett. B 751, 559 (2015).

[36] S. Borsanyi, Z. Fodor, J. N. Guenther, R. Kara, S. D. Katz, P. Parotto, A. Pasztor, C. Ratti, and K. K. Szabo, QCD Crossover at Finite Chemical Potential from Lattice Simulations, Phys. Rev. Lett. 125, 052001 (2020).

[37] S. Mogliacci, J. O. Andersen, M. Strickland, N. Su, and A. Vuorinen, Equation of state of hot and dense QCD: Resummed perturbation theory confronts lattice data, J. High Energy Phys. 12 (2013) 055.

[38] N. Haque, J. O. Andersen, M. G. Mustafa, M. Strickland, and N. Su, Three-loop pressure and susceptibility at finite temperature and density from hard-thermal-loop perturbation theory, Phys. Rev. D 89, 061701(R) (2014).

[39] N. Haque and M. Strickland, Next-to-next-to leading-order hard-thermal-loop perturbation-theory predictions for the curvature of the QCD phase transition line, Phys. Rev. C 103, L031901 (2021).

[40] R. Bellwied, S. Borsanyi, Z. Fodor, S. D. Katz, A. Pasztor, C. Ratti, and K. K. Szabo, Fluctuations and correlations in high temperature QCD, Phys. Rev. D 92, 114505 (2015).

[41] H. T. Ding, S. Mukherjee, H. Ohno, P. Petreczky, and H. P. Schadler, Diagonal and off-diagonal quark number susceptibilities at high temperatures, Phys. Rev. D 92, 074043 (2015).

[42] S. Pratt, E. Sangaline, P. Sorensen, and H. Wang, Constraining the Equation of State of Superhadronic Matter from Heavy-Ion Collisions, Phys. Rev. Lett. 114, 202301 (2015).

[43] S. Borsanyi, G. Endrodi, Z. Fodor, A. Jakovac, S. D. Katz, S. Krieg, C. Ratti, and K. K. Szabo, The QCD equation of state with dynamical quarks, J. High Energy Phys. 11 (2010) 077.

[44] S. Borsanyi, Z. Fodor, C. Hoelbling, S. D. Katz, S. Krieg, and K. K. Szabo, Full result for the QCD equation of state with $2+1$ flavors, Phys. Lett. B 730, 99 (2014).

[45] A. Bazavov et al. (HotQCD Collaboration), Equation of state in $(2+1)$-flavor QCD, Phys. Rev. D 90, 094503 (2014).

[46] J. Gunther, R. Bellwied, S. Borsanyi, Z. Fodor, S. D. Katz, A. Pasztor, and C. Ratti, The QCD equation of state at finite density from analytical continuation, EPJ Web Conf. 137, 07008 (2017).

[47] A. Bazavov et al., Skewness, kurtosis, and the fifth and sixth order cumulants of net baryon-number distributions from lattice QCD confront high-statistics STAR data, Phys. Rev. D 101, 074502 (2020).
[48] W.-j. Fu, X. Luo, J. M. Pawlowski, F. Rennecke, R. Wen, and S. Yin, Hyper-order baryon number fluctuations at finite temperature and density, arXiv:2101.06035.

[49] R. Bellwied, S. Borsanyi, Z. Fodor, J. N. Guenther, J. Noronha-Hostler, P. Parotto, A. Pasztor, C. Ratti, and J. M. Stafford, Off-diagonal correlators of conserved charges from lattice QCD and how to relate them to experiment, Phys. Rev. D 101, 034506 (2020).

[50] J. Noronha-Hostler, P. Parotto, C. Ratti, and J. M. Stafford, Lattice-based equation of state at finite baryon number, electric charge and strangeness chemical potentials, Phys. Rev. C 100, 064910 (2019).

[51] A. Monnai, B. Schenke, and C. Shen, Equation of state at finite densities for QCD matter in nuclear collisions, Phys. Rev. C 100, 024907 (2019).

[52] B. Friman, F. Karsch, K. Redlich, and V. Skokov, Fluctuations as probe of the QCD phase transition and freeze-out in heavy ion collisions at LHC and RHIC, Eur. Phys. J. C 71, 1694 (2011).

[53] C. Ratti, S. Roessner, and W. Weise, Quark number susceptibilities: Lattice QCD versus PNJL model, Phys. Lett. B 649, 57 (2007).

[54] R. Critelli, J. Noronha, J. Noronha-Hostler, I. Portillo, C. Ratti, and R. Rougemont, Critical point in the phase diagram of primordial quark-gluon matter from black hole physics, Phys. Rev. D 96, 096026 (2017).

[55] P. Parotto, M. Bluhm, D. Mroczek, M. Nahrgang, J. Noronha-Hostler, K. Rajagopal, C. Ratti, T. Schäfer, and M. Stephanov, QCD equation of state matched to lattice data and exhibiting a critical point singularity, Phys. Rev. C 101, 034901 (2020).

[56] M. Giordano and A. Pásztor, Reliable estimation of the radius of convergence in finite density QCD, Phys. Rev. D 99, 114510 (2019).

[57] M. Giordano, K. Kapas, S. D. Katz, D. Nogradi, and A. Pasztor, Radius of convergence in lattice QCD at finite $\mu_{B}$ with rooted staggered fermions, Phys. Rev. D 101, 074511 (2020).

[58] S. Mukherjee and V. Skokov, Universality driven analytic structure of QCD crossover: Radius of convergence in baryon chemical potential, Phys. Rev. D 103, 071501 (2021).

[59] See Supplemental Material at http://link.aps.org/ supplemental/10.1103/PhysRevLett.126.232001. Here, we further motivate the approach we pursue in this Letter, by presenting an additional analysis carried out on a cheaper, coarser lattice, as well as a mock analysis addressing the issues of the Taylor expansion. Furthermore, we present the details of the lattice determination of the coefficients $\kappa_{2}^{i j}$ and $\kappa_{4}^{i j}$. We also detail the necessary steps and formulae necessary for the extrapolation of thermodynamic quantities at real chemical potential. Finally, we present analogous analyses for strangeness-related observables.

[60] www.gauss-centre.eu. 